\title{
Nsambya Community Home-Based Care Complements National HIV and TB Management Efforts and Contributes to Health Systems Strengthening in Uganda: An Observational Study
}

\author{
William Massavon, ${ }^{1,2}$ Levi Mugenyi, ${ }^{3}$ Martin Nsubuga, ${ }^{4}$ Rebecca Lundin, ${ }^{1}$ \\ Martina Penazzato, ${ }^{1}$ Maria Nannyonga, ${ }^{2}$ Charles Namisi, ${ }^{2}$ Resty Ingabire, ${ }^{2}$ \\ Daniel Kalibbala, ${ }^{2}$ Susan Kironde, ${ }^{2}$ Paola Costenaro, ${ }^{1}$ Davide Bilardi, ${ }^{1}$ Antonio Mazza, \\ Bart Criel, ${ }^{6}$ James K. Tumwine, $^{7}$ Janet Seeley, $^{8}$ and Carlo Giaquinto ${ }^{1}$ \\ ${ }^{1}$ Department of Paediatrics, University of Padua, Via Giustiniani 3, 35128 Padua, Italy \\ ${ }^{2}$ Home Care Department, Saint Raphael of Saint Francis Hospital (Nsambya Hospital), Kampala, Uganda \\ ${ }^{3}$ Infectious Diseases Research Collaboration, Mulago Hospital Complex, Kampala, Uganda \\ ${ }^{4}$ Saint Raphael of Saint Francis Hospital (Nsambya Hospital), Kampala, Uganda \\ ${ }^{5}$ Santa Chiara Hospital, Via Largo Gold Medals 9, 38122 Trento, Italy \\ ${ }^{6}$ Department of Public Health, Institute of Tropical Medicine, Nationalestraat 155, 2000 Antwerp, Belgium \\ ${ }^{7}$ Department of Paediatrics and Child Health, College of Health Sciences, Makerere University, Kampala, Uganda \\ ${ }^{8}$ MRC/UVRI Uganda Research Unit on AIDS, Plot 51-59, Nakiwogo Road, Entebbe, Uganda
}

Correspondence should be addressed to William Massavon; wmassavon@gmail.com

Received 30 November 2013; Accepted 8 January 2014; Published 6 March 2014

Academic Editors: C. M. Buchalla and R. E. Fullilove

Copyright (C) 2014 William Massavon et al. This is an open access article distributed under the Creative Commons Attribution License, which permits unrestricted use, distribution, and reproduction in any medium, provided the original work is properly cited.

Community Home-Based Care $(\mathrm{CHBC})$ has evolved in resource-limited settings to fill the unmet needs of people living with HIV/AIDS (PLHA). We compare HIV and tuberculosis (TB) outcomes from the Nsambya CHBC with national averages in Kampala, Uganda. This retrospective observational study compared HIV and TB outcomes from adults and children in the Nsambya CHBC to national averages from 2007 to 2011. Outcomes included numbers of HIV and TB patients enrolled into care, retention, loss to follow-up (LTFU), and mortality among patients on antiretroviral therapy (ART) at 12 months from initiation; new smearpositive TB cure and defaulter rates; and proportion of TB patients tested for HIV. Chi-square test and trends analyses were used to compare outcomes from Nsambya CHBC with national averages. By 2011, approximately 14,000 PLHA had been enrolled in the Nsambya CHBC, and about 4,000 new cases of TB were detected and managed over the study period. Overall, retention and LTFU of ART patients 12 months after initiation, proportion of TB patients tested for HIV, and cure rates for new smear-positive TB scored higher in the Nsambya CHBC compared to national averages. The findings show that Nsambya CHBC complements national HIV and TB management and results in more positive outcomes.

\section{Background}

In the wake of the human immunodeficiency virus (HIV) epidemic in Sub-Saharan Africa (SSA), alternative service delivery models like the Community Home-Based Care (CHBC) [1-6] have evolved to fill the gap left by overstretched and underresourced health systems. CHBC includes any form of care (physical, psychosocial, palliative, and spiritual) given to the sick and the affected in their own homes and care extended from the hospital or health facility to their homes through family participation and community involvement $[7,8]$. CHBC provides for the unmet needs of the large and growing population of PLHA in many resource-limited settings $[7,9,10]$. However, the effects of CHBC on national HIV and TB outcomes have not been examined in detail.

In Uganda, the first CHBC programmes were established in 1987 in response to increasing numbers of acutely ill HIV/AIDS patients leading to congestion of hospital 
wards, increased staff workload, and excessive pressure on infrastructure. Three different organisations pioneered this approach: Kitovu Mobile HIV Programme, The AIDS Support Organization (TASO), and Nsambya Hospital Home Care Department, popularly known as Nsambya Home Care (NHC). TASO was started by local people, whereas, Kitovu Mobile and Nsambya Home Care were pioneered by catholic missionary sisters from Ireland.

HIV and TB present important public health problems and health systems challenges for the country. According to the 2011/12 Uganda AIDS Commission Progress Report, Uganda has a generalized HIV epidemic, and prevalence has increased from $6.4 \%$ in 2004 to $7.3 \%$ in 2011 [11]. Uganda is also one of the 22 high-burden countries with respect to TB [12], and the 2012 WHO Global TB Control Report shows that Uganda had a TB prevalence of 183 (95-298) and an incidence rate of 193 (156-234) per 100,000 population, respectively. In the same report, $53 \%$ of $\mathrm{TB}$ patients tested positive for HIV [13]. To deal with the dual epidemics, the ministry of health $(\mathrm{MoH})$ developed national policy guidelines for the integrated management of TB/HIV coinfection. The guidelines aim, among other things, to reduce the burden of both diseases, by improving detection and quality of care and promoting access through decentralization of services to the lower levels of the health systems, where the majority of the population lives [14].

The Ugandan guidelines for scaling up ART [15] outline a primary care approach and make provisions for a $\mathrm{CHBC}$ model, in line with the WHO framework for action on $\mathrm{CHBC}$ in resource-limited settings [7]. This encourages synergies in the implementation of interventions for both HIV and TB. By mandate, $\mathrm{CHBC}$ is expected among other functions to provide palliative care and pain management, implement HIVrelated interventions, and serve as a bridge to extend HIV care, treatment, and psychosocial support services beyond the traditional health facilities to the large and growing population of PLHA in their homes and communities [16]. Clearly, $\mathrm{CHBC}$ is consistent with the designed national response, and as a service delivery model, it could promote integrated management of the dual epidemics in Uganda and other settings with similar problems [17-20].

In this paper, we describe the Nsambya $\mathrm{CHBC}$ and examine the results and their effects on national HIV and TB outcomes and their contribution to health systems strengthening in Uganda. Additionally, we highlight some challenges and recommend practical steps to strengthen implementation of $\mathrm{CHBC}$ in a resource-limited setting.

\section{Methods}

2.1. Study Design, Setting and Population. This retrospective observational study compared HIV and TB outcomes from the Nsambya CHBC to national averages reported by the National TB and Leprosy Programme (NTLP) and the National AIDS Control Programme (NACP) over five years (2007-2011). The study was conducted at St. Raphael of St. Francis Hospital (Nsambya Hospital), Home Care Department in Kampala, Uganda. Nsambya Hospital is a faithbased private-not-for-profit facility owned by the Catholic
Archdiocese of Kampala and accredited by both the ministry of health $(\mathrm{MoH})$ and the Uganda Catholic Medical Bureau (UCMB). It is a general tertiary referral hospital with a bed capacity of 361 and involved in research and training of postgraduate doctors, nurses, midwives, and laboratory technicians.

The study population consisted of adults and children receiving $\mathrm{HIV}$ and $\mathrm{TB}$ care, treatment, and psychosocial support services over the study period.

2.2. Description of $C H B C$. NHC was established to extend basic health services into patients' homes, reduce pressure on hospital workers and infrastructure, and encourage family members to participate in the care of their relatives. This service was also intended to promote early hospital discharge, follow-up after discharge, and community involvement. What started as a team of three health workers providing palliative care to patients in their homes has evolved into a specialized HIV and TB centre. NHC has a catchment area stretching across four districts in and around Kampala and covers approximately $21 \mathrm{~km}$ in radius. The estimated population of the catchment area was about 4 million in 2012 [21]. Over the years, NHC has evolved into a CHBC with the development of community components, which include community engagements, a community-based volunteer programme, community outreach programmes, and outreach clinics.

The Nsambya CHBC is a blend between facility-based care and home-based care with the community serving as an important intermediary. It employs task shifting to overcome some of the shortages in the workforce and uses home visits and outreach clinics to get services closer to patients. In addition, psychosocial support services help patients to deal with some of the challenges posed by HIV positive status and poverty in accessing healthcare in poor-resource settings. The pillars of the CHBC and how they function, patient enrolment practice, tracking of defaulters, and other interventions have been described in detail in a previous study [22].

2.3. Programmes Implemented with $C H B C$. Prior to implementing programmes with the $\mathrm{CHBC}$, donors and partners made concerted efforts to operate within existing national policy guidelines as much as possible. That understanding paved the way for establishing a framework of administrative and operational integration among donors and partners aimed at coordinating resources, promoting efficiency, and avoiding measures that could potentially damage the health system.

Within that framework, several closely related programmes were implemented with the CHBC: HIV prevention education, counselling and testing, ART, HIV chronic and palliative care, TB treatment, Intensified TB Case Finding (ICF), and Isoniazid Preventive Therapy (IPT). The programmes were vertical owing to the weak state of the general health system, and the approach can be considered as a contextualized solution [23-26]. Nonetheless, the Nsambya $\mathrm{CHBC}$ has extensive and important functional linkages to the 
general health system and all the relevant stakeholders. For instance, the $\mathrm{MoH}$ supports the TB clinic within the Nsambya $\mathrm{CHBC}$ to function as a national referral facility that provides TB treatment for the public and participates in surveillance activities and national TB-HIV studies. The various interrelationships of the Nsambya CHBC are illustrated in Figure 1, and the key players, processes, and linkages to the observed outcomes are summarized in Figure 2.

The Nsambya CHBC was funded mainly by nongovernmental initiatives through a long-standing faith-based solidarity, and minimal support from the MoH. The faithbased solidarity also provided vital technology and technical assistance to achieve a common goal. The goal was to provide comprehensive HIV care, treatment, and psychosocial support services for HIV-infected patients and their families and affected communities. Services were generally free of charge; however, adults paid a user fee of 1,000 Uganda shillings, the equivalent of 38 cents of a US dollar at the time of this study, per visit.

2.4. Data Collection. Data from routine programme activities, programme reports, patients' records, and HIV and TB registers at $\mathrm{NHC}$ were collected for the study. Country-level data were obtained from the NACP and NTLP reports as well as from global HIV and TB reports. Some of the data were incomplete from the three institutions in the study. Consequently, the analyses were limited to periods with complete data, and that has been provided under Section 3.

2.5. Statistical Methods and Data Analysis. Primary study outcomes included the proportions of ART patients retained in care, LTFU and mortality at 12 months from ART initiation, proportion of TB patients tested for HIV, and cure and defaulter rates for new smear-positive cases. Secondary outcomes included HIV-TB coinfection and ART status among defaulters and bed occupancy rate for HIV-related hospital admissions within 12 months of starting the CHBC. Bed occupancy rate was determined from a hospital report (unpublished).

The data were analyzed with Microsoft Excel programme version 2010 and STATA version 12. Chi-square tests were used to determine the differences and trends between the mean outcomes from the Nsambya CHBC and national outcomes. In addition, chi-square test and Fisher's exact test were used to determine differences in the proportions of TB defaulters coinfected with HIV, not coinfected, receiving ART, and not on ART.

The Uganda National Council for Science and Technology granted ethical approval for the study (UNCST Ref: HS 1383). The relevant authorities waived informed consent.

\section{Results}

3.1. Effect on HIV Patient Outcomes. It is estimated that about 14,000 PLHA and their families have been enrolled in the Nsambya CHBC since its inception in 1987. Overall, about $91.6 \%$ were adults, $67.7 \%$ were females, and $8.4 \%$ were children. From January 2009 to December 2011, on average,

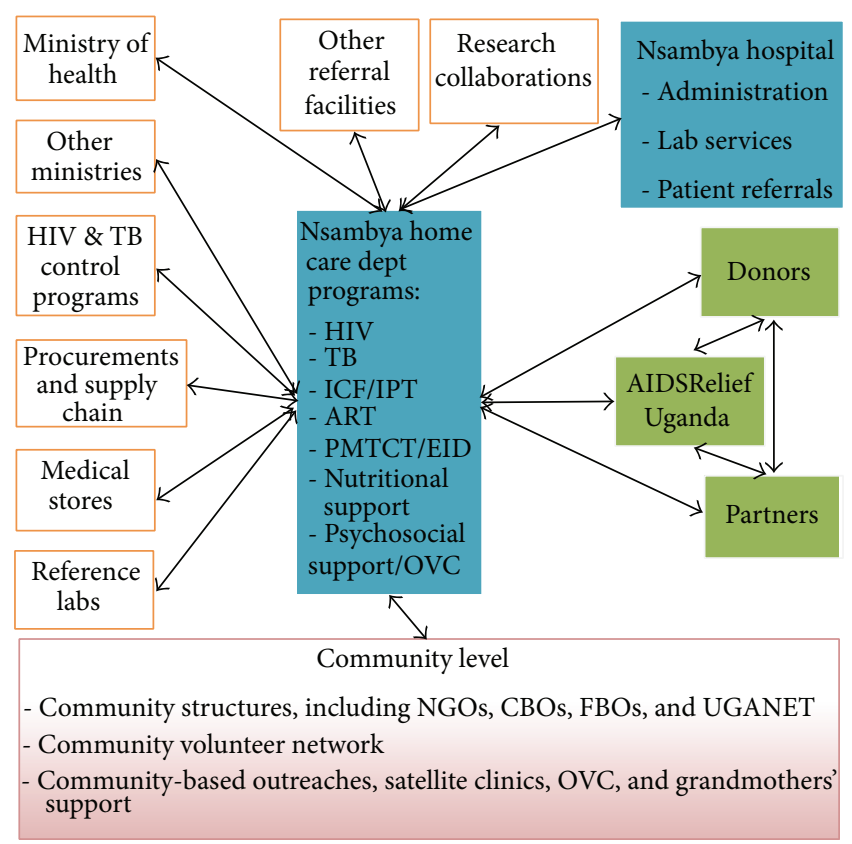

Figure 1: Nsambya Community Home-Based Care and linkages to the general health system, stakeholders, and communities. NGO: nongovernmental organization; $\mathrm{CBO}$ : community-based organizations; FBO: faith-based organizations; UGANET: Uganda network on law, ethics, and HIV/AIDS; OVC: orphans and vulnerable children; TB: tuberculosis; ICF/IPT: intensified case finding and Isoniazid preventive therapy; ART: antiretroviral therapy; PMTCT: prevention of mother to child transmission; EID: early infant diagnosis.

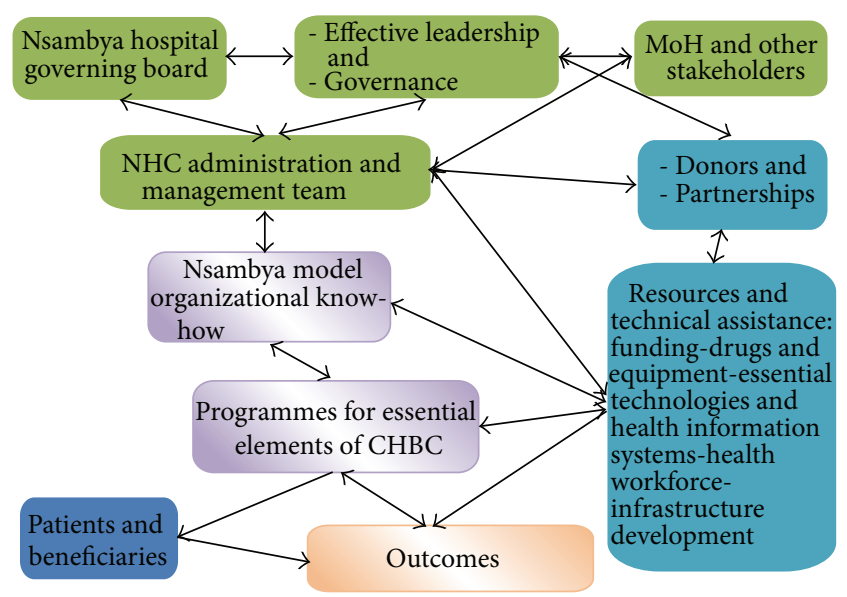

FIGURE 2: Illustration of the key players in the Nsambya CHBC model, the vital components, and linkages to outcomes. Conceptual framework of the Nsambya CHBC, the context within which it operates, and functional connections to all stakeholders including beneficiaries.

$90 \%(89 \%-91 \%)$ of the Nsambya CHBC patients on ART were retained in care, 12 months after ART initiation, compared to $83.3 \%(83.2 \%-83.4 \%)$ for the national average. The difference was significant $\left(\chi^{2}=60.3, P<0.001\right)$ and the trends were significantly different $\left(\chi^{2}\right.$ for trend $\left.=26.8, P<0.001\right)$, 


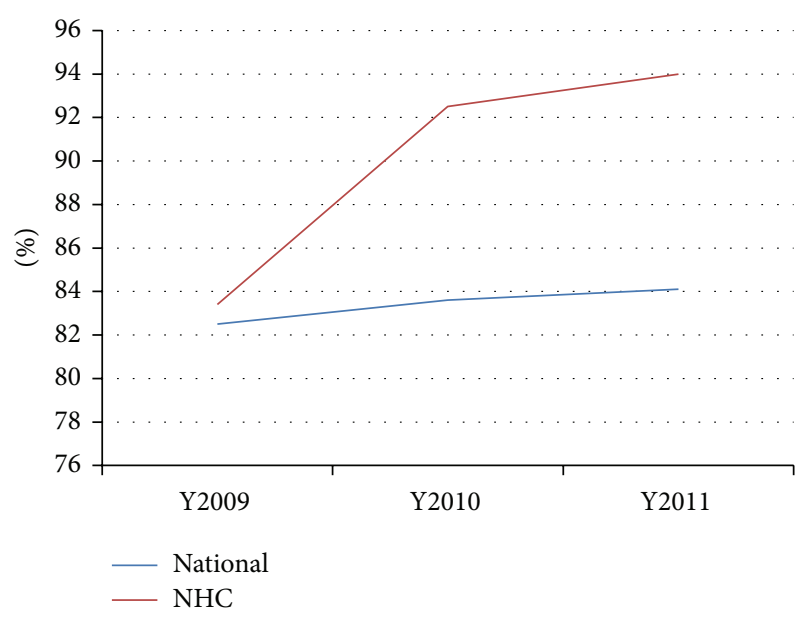

FIGURE 3: Trends in retention in care (12 months after ART initiation): Nsambya CHBC versus National (2009-2011). $\mathrm{NHC}=\mathrm{NCBHC}$; $\mathrm{Y}$ - on $x$-axis of the graph represents year.

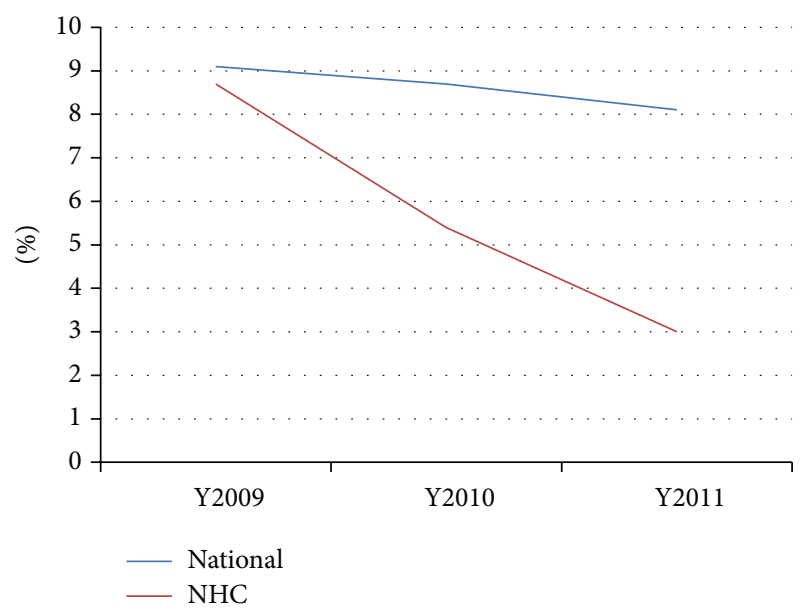

Figure 4: Trends in Loss-to-follow-up: Nsambya CHBC versus National (12 months after ART initiation), 2009-2011. $\mathrm{NHC}=\mathrm{NCHBC}$; $\mathrm{Y}$ - on $x$-axis represents year.

with the Nsambya CHBC having more positive trends than national (Table 1 and Figure 3).

The Nsambya CHBC recorded an average LTFU rate of $5.7 \%(4.9 \%-6.4 \%)$ for ART patients 12 months after initiation, compared to the national figure of $8.7 \%(8.6 \%-$ $8.8 \%)$. Overall, LTFU differed significantly $\left(\chi^{2}=95.8, P<\right.$ 0.001 ), as well as the trends ( $\chi^{2}$ for trend $=95.7, P<0.001$ ), with the Nsambya CHBC having a significantly decreasing trend compared to the national trend (Table 1 and Figure 4).

The proportion of ART patients that died, 12 months after ART initiation, was 5.4\% (4.7\%-6.1\%) for the Nsambya $\mathrm{CHBC}$ and $4.6 \%(4.5 \%-4.7 \%)$ for the national figure. Overall, the difference in mortality $\left(\chi^{2}=38.9, P<0.001\right)$ and the trends were significant $\left(\chi^{2}\right.$ for trend $\left.=35.8, P<0.001\right)$. However, the trend was significantly decreasing under the Nsambya CHBC compared to the National (Table 1 and Figure 5).

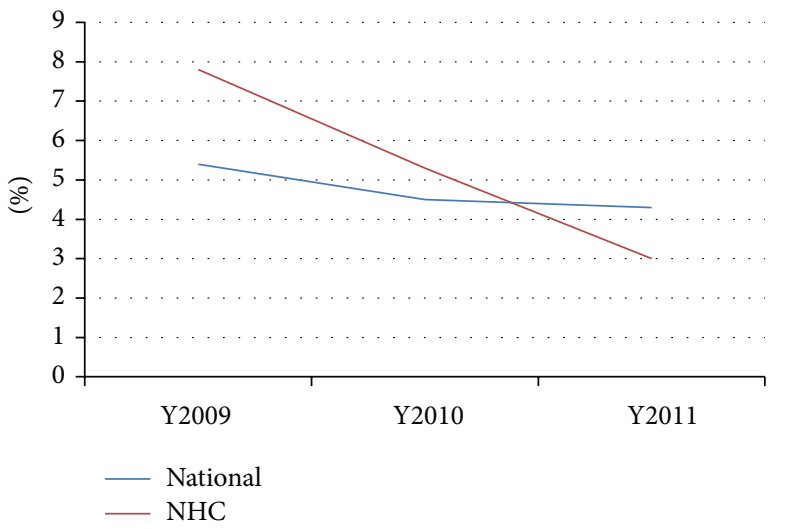

FIgURE 5: Trends in mortality at 12 months after ART initiation: Nsambya CHBC versus National (2009-2011). NHC=NCHBC; Yon $x$-axis represents year.

3.2. Effect on TB Patient Outcomes. Approximately 4,000 new TB cases were detected and managed from 2007 to 2011. Adults constituted $92.3 \%$, females $51.0 \%$, and children $7.7 \%$ of the cases. On average, $95 \%$ of TB patients from the Nsambya CHBC were tested for HIV as against $72 \%$ for the national value. From 2007 to 2010, the Nsambya CHBC recorded an average cure rate of $54.6 \%$ for new smear-positive TB patients, while the figure for the national average was $30.8 \%$. The difference was significant $\left(\chi^{2}=21.2, P=0.001\right)$, but there was no difference in the trends $\left(\chi^{2}\right.$ for trend $=3.0$, $P=0.083$, Table 2 and Figure 6). New smear-positive TB defaulter rates were $10.1 \%$ and $10.7 \%$ for the Nsambya CHBC and National, respectively. The difference was not significant $\left(\chi^{2}=2.1, P=0.541\right)$ and the trends did not differ $\left(\chi^{2}\right.$ for trend $=0.02, P=0.877)$, Table 2 and Figure 7 .

Overall, there were $110 \mathrm{~TB}$ defaulters, $54.5 \%$ (60/110) were enrolled in care in the Nsambya CHBC and the rest were referrals from other facilities. Majority of the TB defaulters were HIV-TB coinfected $(72 \%, P<0.001)$ and included all the TB defaulters enrolled in care in the Nsambya CHBC, who accounted for $76 \%(P<0.001)$ of all the HIV-TB coinfected defaulters. Overall, minority of the TB defaulters were receiving ART (39\%), and when stratified by source of patients, the proportions were similar: $38 \%$ versus $42 \%$ ( $P=$ $0.769)$ for Nsambya CHBC patients and referrals respectively (Table 3).

3.3. Effect on Bed Occupancy. The immediate impact of the Nsambya CHBC was a remarkable reduction in bed occupancy from an average of three months to two weeks for HIV/AIDS-related hospital admissions in 1987, within 12 months of starting the CHBC, long before ART became publicly accessible in the country (data not presented).

\section{Discussion}

Overall, the core findings from this study demonstrate that the Nsambya CHBC complements national HIV and TB management and resulted in a higher proportion of ART patients retained in care and a lower LTFU rate, 12 months 
TABLE 1: Comparison of HIV treatment outcomes from the Nsambya CHBC with national averages, 12 months after ART initiation, Kampala, Uganda, (2009-2011).

\begin{tabular}{|c|c|c|c|c|}
\hline Year & National \% $(n / N)$ & Nsambya CHBC \% $(n / N)$ & Overall chi-square $P$ value & Chi-square for trend $P$ value \\
\hline \multicolumn{5}{|c|}{ Retained in care } \\
\hline 2009 & $82.5(252155 / 305642)$ & $83.4(2946 / 3532)$ & & \\
\hline 2010 & $83.6(310435 / 371334)$ & $92.5(4352 / 4705)$ & $<0.001$ & $<0.001$ \\
\hline 2011 & $83.9(386693 / 460898)$ & $94.0(5185 / 5516)$ & & \\
\hline \multicolumn{5}{|c|}{ Loss to follow-up } \\
\hline 2009 & $9.1(27813 / 305642)$ & $8.7(307 / 3532)$ & & \\
\hline 2010 & $8.7(32306 / 371334)$ & $5.4(254 / 4705)$ & $<0.001$ & $<0.001$ \\
\hline 2011 & $8.2(37794 / 460898)$ & $3.0(166 / 5516)$ & & \\
\hline \multicolumn{5}{|c|}{ Mortality } \\
\hline 2009 & $5.4(16505 / 305642)$ & $7.8(276 / 3532)$ & & \\
\hline 2010 & $4.5(16710 / 371334)$ & $5.3(249 / 4705)$ & $<0.001$ & $<0.001$ \\
\hline 2011 & $3.9(17975 / 460898)$ & $3.0(166 / 5516)$ & & \\
\hline
\end{tabular}

TABLE 2: Comparison of new smear-positive TB cure and defaulter rates from the Nsambya CHBC with national averages, Kampala, Uganda, (2007-2010).

\begin{tabular}{|c|c|c|c|c|}
\hline Year & National \% $(n / N)$ & Nsambya CHBC \% $(n / N)$ & Overall chi-square $P$ value & Chi-square for trend $P$ value \\
\hline \multicolumn{5}{|c|}{ New smear-positive TB cure rates } \\
\hline 2007 & $30.7(6540 / 21303)$ & $35.6(99 / 278)$ & \multirow{4}{*}{0.001} & \multirow{4}{*}{0.083} \\
\hline 2008 & $27.5(6261 / 22766)$ & $56.1(165 / 294)$ & & \\
\hline 2009 & $30.0(6934 / 23113)$ & $55.8(168 / 301)$ & & \\
\hline 2010 & $34.9(8186 / 23456)$ & $71.0(171 / 241)$ & & \\
\hline \multicolumn{5}{|c|}{ New smear-positive TB defaulter rates } \\
\hline 2007 & $15.1(3217 / 21303)$ & $14.0(39 / 278)$ & \multirow{4}{*}{0.541} & \multirow{4}{*}{0.877} \\
\hline 2008 & $11.2(2550 / 22766)$ & $11.2(33 / 294)$ & & \\
\hline 2009 & $11.5(2658 / 23113)$ & $8.3(25 / 301)$ & & \\
\hline 2010 & $5.1(1196 / 23456)$ & $7.0(17 / 241)$ & & \\
\hline
\end{tabular}

TABLE 3: Comparison of TB defaulters (adults and children) receiving treatment in the Nsambya CHBC by HIV-TB coinfection and ART status, Kampala (2007-2010).

\begin{tabular}{|c|c|c|c|c|}
\hline Characteristic & $\begin{array}{c}\text { Referred patients } N=50 \\
n(\%)\end{array}$ & $\begin{array}{c}\text { NCHBC patients } N=60 \\
n(\%)\end{array}$ & Total $N=110$ & Fisher's exact/chi-square-tests $P$ value \\
\hline \multicolumn{5}{|c|}{ HIV-TB coinfected } \\
\hline Yes & $19(38)$ & $60(100)$ & $79(72)$ & \multirow{2}{*}{$<0.001^{*}$} \\
\hline No/unknown & $31(62)$ & $0(0)$ & $31(28)$ & \\
\hline \multicolumn{5}{|c|}{ HIV-TB coinfected on ART } \\
\hline Yes & $8(42)$ & $23(38)$ & $31(39)$ & \multirow{2}{*}{$0.769^{* *}$} \\
\hline No & $11(58)$ & $37(62)$ & $48(61)$ & \\
\hline
\end{tabular}

${ }^{*}$ Fisher's exact test, ${ }^{* *}$ Chi-square test.

Data sources.

(1) Global AIDS Response Progress Report, Uganda AIDS Commission April 2012, Kampala.

(2) Status of Antiretroviral Therapy Services in Uganda. Quarterly ART Reports for October-December 2010.

(3) Status of Antiretroviral Therapy Services in Uganda. Quarterly ART Reports for January-June 2011.

(4) Status of Antiretroviral Therapy Services in Uganda. Quarterly ART Reports for October-December 2011.

(5) CDC Uganda Quarterly Reports; October-December 2009.

(6) CDC Uganda Quarterly Reports; October-December 2010.

(7) CDC Uganda Quarterly Reports; October-December 2011.

(8) Annual Health Sector Performance Report. Financial Years 2009/10, Ministry of Health, Uganda, Kampala

(9) Annual Health Sector Performance Report. Financial Years 2010/11, Ministry of Health, Uganda, Kampala.

(10) WHO Report 2011: Global TB Control.

(11) WHO: TB_notification_2012-06-11.csv.

(12) WHO: TB_outcomes_2012-06-15.csv, http://www.who.int/tb/country/data/download/en/index.html.

(13) Nsambya Home Care TB registers. 


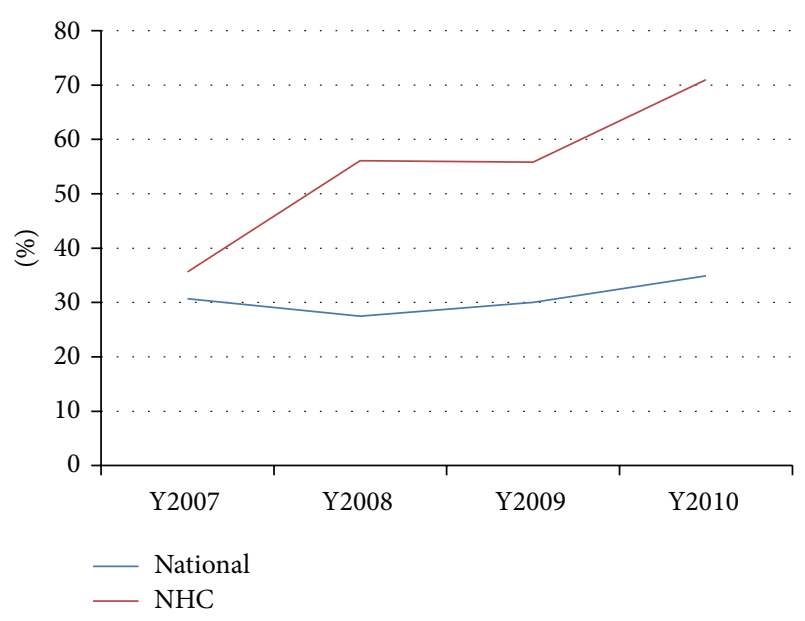

Figure 6: Trends in new smear-positive TB cure rates: Nsambya CHBC versus National (2007-2010). NHC $=$ NCHBC; Y- on $x$-axis represents year.

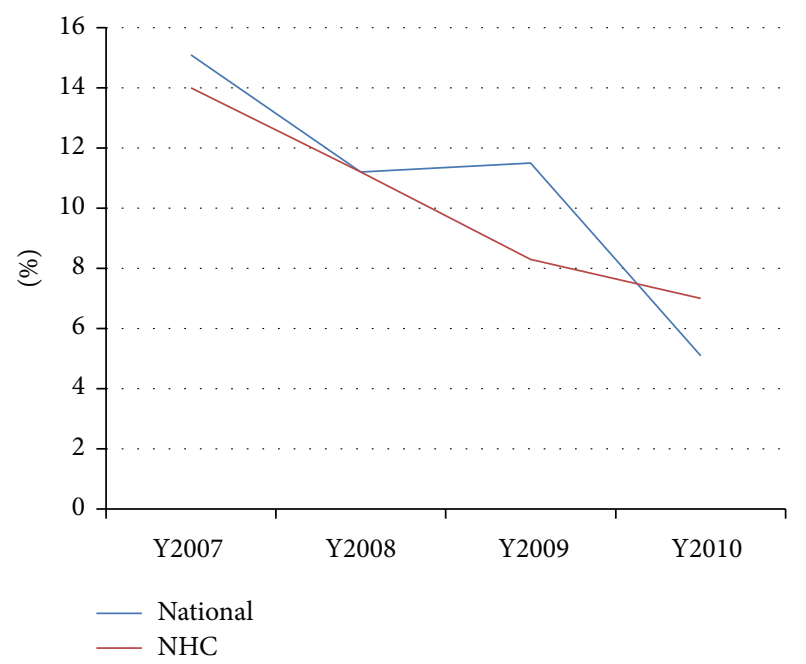

FIgURE 7: New smear-positive TB defaulter trends: Nsambya CHBC versus National (2007-2010). NHC=NCHBC; Y- on $x$-axis represents year.

after initiation. We believe the higher retention in care and lower LTFU rates seen among the ART patients could be revealing the results of 25 years of evolution of the Nsambya $\mathrm{CHBC}$, from preparing PLHA for death in the pre-ART era to keeping them alive through ART and long-term followup measures. The process entailed regular review of CHBC design to make it sensitive to some key challenges faced by patients while seeking health care. That translated into additional psychosocial support services such as the OVC support programme, food supplements to help with food insecurity, economic empowerment, particularly of adolescents through sponsorships for vocational trainings, and some caregivers to enable them to deal with poverty and other negative impacts of HIV/AIDS [22]. Other factors include strategies such as tracking of defaulting patients, community involvements, task shifting to community volunteers, nurses, counselors, and social workers [27], and using outreaches to promote geographical access to some services. Furthermore, the evolutionary process involved the adoption of measures that have contributed to health system strengthening, as a stronger health system [5] is crucial for effective service delivery.

We think that the slightly higher mortality rate seen among Nsambya CHBC patients on ART might be due to improved tracking of patients considered "lost to follow-up." Indeed, a variable proportion of ART patients labelled as "lost to care" were actually dead upon tracking, and that observation is consistent with the literature [28-30]. Our mortality rate may also be reflecting improved documentation and reporting of deaths from the communities by community volunteers, who are residents of the communities. Overall, the mortality trend was reducing much more in the Nsambya $\mathrm{CHBC}$, as depicted in Figure 5.

We also found that a higher percentage of TB patients were tested for HIV in the Nsambya CHBC and the average cure rate for new smear-positive TB patients was higher than the national average. However, the defaulter rates were similar. Various factors may explain the higher proportion of TB patients tested for HIV and the improved TB cure rates in the Nsambya CHBC. The Nsambya CHBC has a TB clinic and a laboratory for various tests including sputum microscopy on the same premises as the main HIV clinic. That structural arrangement coupled with training of health workers on policy guidelines for the integrated management of HIV-TB coinfection may have strengthened management of the two diseases. That arrangement may also have raised awareness among health workers and patients as well as facilitated screening of TB patients for HIV and vice versa. Moreover, patients see the arrangement as convenient and cost-saving to have HIV and TB screening and treatment at the same facility [31]. The high TB defaulter rate was unexpected, particularly when compared to LTFU among ART patients in the Nsambya CHBC. Nevertheless, that finding might be linked to the overall small proportion of TB defaulters started on ART (Table 3), which is in keeping with the literature $[32,33]$. In addition, TB patients referred to receive treatment but not enrolled in the Nsambya CHBC could not be tracked because of disjointed health information systems and logistic challenges. This observation could be reflecting a wider problem and calls for early initiation of ART in all HIV-TB coinfected patients in line with the recent revisions of the treatment guidelines $[34,35]$ and concerted efforts to track all $\mathrm{TB}$ patients receiving treatment in the Nsambya CHBC.

The remarkable reduction in bed occupancy was feasible because of early discharge from hospital, home-based care provided by outreach staffs, and support from family members and friends of patients and community involvements. Studies from Uganda $[36,37]$ and elsewhere in SSA $[38,39]$ reported high HIV-related hospital admissions, sometimes to the exclusion of non-HIV patients in the 1990s and early 2000s. Undoubtedly, the reduction in bed occupancy has multiple impacts, such as freeing up beds for non-HIV patients, decreased workload on health workers and reduced pressures on health infrastructure, all of which have gains for the health system [26]. There may also be gains for the 
patients and their caregivers from the shorter stays, such as overall costs. With the advent of ART, the reduction in bed occupancy has been sustained, as the health conditions of many HIV patients improved and relatively fewer patients were admitted to hospital and for shorter durations, in line with the literature [40-42]. Over the years, community involvements seem to have raised awareness about HIV and reduced stigma to some extent, as reported by some patients and their caregivers as well as community volunteers, some of whom are expert patients. These positive effects could contribute to the building blocks for chronic disease management in resource-limited settings, particularly, where the default healthcare delivery models were not designed for chronic disease management [16].

We have observed some of the positive impacts of home visits and the various forms of psychosocial support, including lessons on self-management on patient outcomes, and believe they could go beyond HIV and TB management to benefit patients with other chronic conditions such as diabetes and hypertension, to mention a few [43]. Yet, that scenario may be dictated by additional funding and other resources to scale up, policy guidelines for regulation, and the political commitment to support and sustain the approach as well as a change in the mind-sets of programme coordinators and managers. Currently, CHBC is largely a "donor-partner" funded initiative operating on a miniature scale $[8,44]$, compared to the existing HIV and TB disease burdens and unmet needs.

4.1. Impact of Long Standing Faith-Based Solidarity. To a large extent, the findings from the Nsambya CHBC illustrate what could be achieved when a common goal is backed by some form of "solidarity", in this case, "a complex and powerful long standing faith-based solidarity" involving international donor-partnerships and local partners. To accomplish the common goal for the solidarity, a wide range of resources and several programmes were envisaged. Somehow, the donors and partners directly or indirectly supported most of the essential pillars of health system strengthening [45, 46] through funding, drugs, equipment, materials, infrastructure development, and vital technologies like electronic databases to support health information systems. The donorpartnership synergies also provided technical assistance to build and maintain systems: train, supervise, monitor and evaluate performance against set standards periodically, thus gradually developing some of the needed systemic capacities [47] as well as a viable service delivery mechanism over time. It is estimated that, at the time of this study, the existing key donors and partners had each supported the Nsambya CHBC for at least eight years covering different periods or with some overlap, and the oldest partner for over 25 years and ongoing. This "longevity of faith-based solidarity" has been pivotal to the survival, evolution, and expansion of the Nsambya CHBC.

4.2. Administrative and Operational Integration. One of the important achievements of the Nsambya CHBC was the establishment of administrative and operational integration among donors and partners. The measures allow some resources to be pooled together, utilized after collective decisions, and accounted for in a transparent manner. Administrative and operational integration has facilitated and somehow harmonized implementation of some common policy guidelines. For instance, there are common policy guidelines for human resources for health management in place. In practice, they translate into common procedures for advertising vacancies, standardized selection criteria, and the use of Ugandan national salary scales. This avoids disparities in salaries and working conditions for health workers with similar qualifications and experiences, but working on different programmes. The measures have reduced duplication, minimized wastage and administrative costs and poaching of health workers, and seem to have promoted efficiency and synergies with better programme outcomes.

4.3. Positive "Spill Over" Effects. Even though the original goal of the Nsambya CHBC was to provide care, treatment, and psychosocial support for PLHA and their families, with time, the additional resources from the HIV programmes appear to have benefited other programmes and the general health system. Notably, TB control, nutritional support for children, and OVC support including sponsorships for vocational training and support for caregivers were benefited [22]. These positive "spill over" effects are consistent with findings from studies in Ethiopia and Malawi [48] and elsewhere $[25,26,49]$ globally.

4.4. Challenges to Be Addressed. Despite the achievements of the Nsambya CHBC, some important challenges remain, and they can be viewed from the level of CHBC, from that of the implementing organization, donor-partner demands and preferences, and the general health system. Documentation and data capturing from community activities need to improve in order to contribute to operational research in the future. The referral networks linking the communities to the outreach clinics and to the department and hospital require strengthening in order to be effective. Community volunteers play vital roles in the referral networks, but they may not be adequately resourced to function effectively. Budgeting must also be clear and stable in order for programmes to be designed in a feasible manner and implemented consistently. This can be difficult to manage with multiple donors and partners providing varying portions of funds over varying periods. Supplies such as drugs and medical products are also received on a variable basis from a range of sources. In order to prevent waste and actively identify areas of both overage and shortage, efficient and timely recording systems for supplies are essential.

Although the Nsambya CHBC has an organizational know-how in place that could be extended to benefit other health problems other than HIV and TB, some donors and partners prefer to fund only specific aspects of the programmes. That state of affairs creates some difficulties among the donor-partner relationships and somehow does not contribute to the realization of the full potentials of CHBC. With respect to the general health system, referral networks are generally fragile, operational guidelines lack visibility, and the disjointed nature of health information 
systems makes it a daunting task to track patients lost to care, especially when they relocate to different cities, towns, or villages.

We recommend the following steps for the relevant authorities to consider in strengthening and expanding implementation of CHBC programmes:

(i) government cofunding and political commitment to scale up CHBC and ensure continuity of support in the face of changes in the donor-partner relationships,

(ii) streamlining the existing patient tracking system to make it sensitive for tracking all patients in care in the Nsambya CHBC,

(iii) strengthening of the referral networks through national guidelines and resource allocation as well as research, and

(iv) a critical assessment of how the CHBC models impact on the general health system.

4.5. Limitations of Study. Potential limitations of this study include issues with documentation (incomplete data), availability, and data quality. Consequently, we believe the reported number of patients ever enrolled in the Nsambya $\mathrm{CHBC}$ could be an underestimation, possibly due to missing data from worn out paper-based registers, before electronic databases became available. To deal with missing data, we relied on reported data from the NACP and NTLP, presented in global HIV and TB reports for the comparisons, whenever possible. That meant that only periods with available reported data could be compared. These limitations were accommodated for by providing the periods for the various analyses in the text under results.

Data on the average bed occupancy rate was from a secondary source (unpublished hospital report) which did not provide the standard deviation for the mean reported. In addition, portions of the relevant paper-based registers for 1987-88 hospital admissions have worn out over the years, resulting in missing data. Therefore, the primary data could not be accessed for analyses.

We also recognize that the national averages level off diversity in data and their sources and therefore believe that, the observed differences in outcomes may not be solely due to the CHBC approach but possibly some other factors, which we were unable to explore.

\section{Conclusions}

We conclude that the Nsambya CHBC complements national $\mathrm{HIV}$ and TB management efforts and resulted in more positive results for several HIV and TB outcomes, when compared to the national averages. The findings could be reflecting the results of 25 years of evolution of the Nsambya $\mathrm{CHBC}$, from preparing PLHA for death in the pre-ART era, to keeping them alive through life-prolonging ART and long-term follow-up measures. This is a process that entailed regular review of the approach, community involvements and additional interventions to mitigate some of the negative impacts of HIV/AIDS, while adopting measures and strategies that have contributed to health system strengthening in the country. This approach may hold the potential for chronic disease management in resource-limited settings. Scaling up $\mathrm{CHBC}$ could have wider positive impacts on the management of not only HIV and TB, but also other chronic diseases as well as the general health system. A complex and powerful long-standing "faith-based solidarity" among international donors and partners has been pivotal to the survival and evolution of the Nsambya CHBC.

\section{Conflict of Interests}

The authors declare that they have no competing interests.

\section{Authors' Contribution}

William Massavon conceived the study and participated in the drafting and editing of the paper, data analysis, and interpretation of the findings. Maria Nannyonga, Susan Kironde, and Resty Ingabire participated in the drafting of the paper. Martina Penazzato and Charles Namisi participated in the drafting and editing of the paper and interpretation of findings. Martin Nsubuga, Paola Costenaro, Davide Bilardi, and Antonio Mazza participated in the editing of the manuscript. Levi Mugenyi and Daniel Kalibbala participated in the data analysis and interpretation of the findings. Rebecca Lundin, Bart Criel, Janet Seeley, Carlo Giaquinto and James K. Tumwine participated in the editing of the paper and interpretation of the findings. All authors read and approved the final paper.

\section{Acknowledgments}

Rev. Sr. Dr. Miriam Duggan started the "Home-Based Care Service" (now CHBC) at Nsambya Hospital in June 1987, and the authors are grateful for the foundation she laid. The authors thank Noerine Kaleeba, cofounder of TASO, and Rev. Sr. Ursula Sharpe, founder of Kitovo Mobile HIV Programme, for their inputs on the history of CHBC in Uganda. The authors thank Dr. J. F. Imoko of the NTLP at the WHO office in Kampala for assisting them with data and expertise. The Nsambya CHBC has been supported by various donors and partners since its inception, thus enabling it to survive difficult times and expand over the years. The authors will always be grateful to all the donors and partners, both in past and present. The authors thank all the patients and families enrolled at Nsambya Home Care, for without them, this $\mathrm{CHBC}$ would not exist. The authors appreciate the support offered by the management of Nsambya Hospital and the Home Care department. The data unit, the social workers' unit, the community volunteers, and the counsellors all contributed to this study, in one way or another. In particular, the authors wish to acknowledge the following staff: Jane Chantal Nakachwa, Fred Kaija, and Lillian Zimula Nannyondo, whose long services have made them the "living institutional memories"; the authors appreciate their dedication and loyalty. Without the inputs of some other staffs this study would 
not have materialized and the authors are grateful to Agnes Alowo, Allen Victor Nagawa, Dan Kimbowa, Isaac Musoke, Francis Sozzi, Maria Kanyesigye, Jamilla Namaala, Christine Namutebi, RoseMary Alwenyi, Grace Anzoyo, and Brian Kawere. The authors appreciate the support of the secretary of the department (Susan Nakayiga) and the hospital and indeed all the staff. Finally, the authors thank the Provincia Autonoma di Trento and Regione Trentino Alto Adige for funding NHC since 2006 through Casa Accoglienza alla Vita Padre Angelo, and the PENTA Foundation for supporting the interventions.

\section{References}

[1] UNAIDS, "UNAIDS report on the global AIDS epidemic," Global Report, 2010, http://www.who.int/hiv/pub/global_report2010/en/.

[2] WHO, "Taking stock: health worker shortages and the response to AIDS," 2006, http://www.who.int/hiv/toronto2006/takingstockttr.pdf.

[3] WHO, "Working together for health," World Health Report, 2006, http://www.who.int/whr/2006/whr06_en.pdf.

[4] E. Friedman, I. Katz, E. Kiley, E. Williams, and A. Lion, "Global Fund's Support for Health Systems Strengthening Interventions: a reference guide," Health Systems 20/20 Project, Physicians for Human Rights, Bethesda, Md, USA, 2011, http://www .healthsystems2020.org/.

[5] A. Buvé, S. Kalibala, and J. McIntyre, "Stronger health systems for more effective HIV/AIDS prevention and care," International Journal of Health Planning and Management, vol. 18, no. 1, pp. S41-S51, 2003.

[6] D. Maher, L. Smeeth, and J. Sekajugo, "Health transition in Africa: practical policy proposals for primary care," Bulletin of the World Health Organization, vol. 88, no. 12, pp. 943-948, 2010.

[7] WHO, "Community home-based care in resource-limited settings: a framework for action," 2002, http://www.who.int/ chp/knowledge/publications/comm_home_based_care/en/index.html.

[8] N. Mohammad and J. Gikonyo, "Operational Challenges Community Home Based Care (CHBC) for PLWHA in Multi-Country Aids Programs (MAP) in Africa," Africa Region Working Paper Series 88, 2005.

[9] UNAIDS, "Keeping the promise: summary of the declaration of commitment on HIV/AIDS," United Nations General Assembly Special Session on HIV/AIDS, New York, NY, USA, 2001, http://whqlibdoc.who.int/unaids/2002/9291731900_eng.pdf.

[10] B. P. Ncama, "Models of community/home-based care for people living with HIV/AIDS in Southern Africa," Journal of the Association of Nurses in AIDS Care, vol. 16, no. 3, pp. 33-40, 2005.

[11] Ministry of Health Uganda, "Annual health sector performance report," Tech. Rep., 2011-2012, http://health.go.ug/docs/ AHSPR_11_12.pdf.

[12] WHO, “The Global Plan to StopTB, 2011-2015,” 2011, http:// www.who.int/tb/publications/global_plan_to_stop_tb/en/.

[13] WHO, “Global tuberculosis report," Tech. Rep., 2012, https:// extranet.who.int/tme/assets/documents/TB12_Report_low.pdf.

[14] Ministry of Health Uganda, "National policy guidelines for Tb/Hiv collaborative activities in Uganda," 2006, http://www .who.int/hiv/pub/guidelines/uganda.pdf.
[15] Ministry of Health Uganda, "National antiretroviral treatment and care guidelines for adults, adolescents, and children," 2008, http://www.who.int/hiv/amds/uganda_moh_treatment_ guidelines.pdf.

[16] UAC, 2007, Moving Toward Universal Access: National HIV \& AIDS Strategic Plan 2007/8, 2011/12, Uganda AIDS Commission, Republic of Uganda http://siteresources.worldbank.org/INTHIVAIDS/Resources/375798.

[17] S. Miti, V. Mfungwe, P. Reijer, and D. Maher, "Integration of tuberculosis treatment in a community-based home care programme for persons living with HIV/AIDS in Ndola, Zambia," International Journal of Tuberculosis and Lung Disease, vol. 7, no. 9, supplement 1, pp. S92-S98, 2003.

[18] F. Terris-Prestholt, L. Kumaranayake, R. Ginwalla et al., "Integrating tuberculosis and HIV services for people living with HIV: costs of the Zambian ProTEST Initiative," Cost Effectiveness and Resource Allocation, vol. 6, article 2, 2008.

[19] J. Uwimana, C. Zarowsky, H. Hausler, and D. Jackson, "Training community care workers to provide comprehensive TB/ HIV/PMTCT integrated care in KwaZulu-Natal: lessons learnt," Tropical Medicine and International Health, vol. 17, no. 4, pp. 488-496, 2012.

[20] J. C. Brust, N. S. Shah, M. Scott et al., "Integrated, home-based treatment for MDR-TB and HIV in rural South Africa: an alternate model of care," The International Journal of Tuberculosis and Lung Disease, vol. 16, no. 8, pp. 998-1004, 2012.

[21] Uganda Bureau of Statistics, "Statistical abstract," 2012, http:// www.ubos.org/onlinefiles/uploads/ubos/pdf\%20documents/ 2012StatisticalAbstract.pdf.

[22] W. Massavon, R. Lundin, P. Costenaro, M. Penazzato, P. C. Namisi et al., "Attrition and loss to follow-up among children and adolescents in a community home-based care HIV programme in uganda," Pediat Therapeut, vol. 3, p. 183, 2013.

[23] B. Criel, G. Kegels, and P. Van Der Stuyft, "Editorial: a framework for analysing the relationship between disease control programmes and basic health care," Tropical Medicine and International Health, vol. 9, no. 6, pp. A1-A4, 2004.

[24] B. Keugoung, J. Macq, A. Buvé, J. Meli, and B. Criel, “The interface between health systems and vertical programmes in Francophone Africa: the managers' perceptions," Tropical Medicine and International Health, vol. 16, no. 4, pp. 478-485, 2011.

[25] W. Van Damme, M. Pirard, Y. Assefa, and J. Van Olmen :, "Which health systems for disease control? How can disease control programmes contribute to health systems strengthening in SubSaharan Africa?" Studies in Health Services Organisation \& Policy 1, Institue of Tropical Medicine, Antwerp, Belgium, 2010, www.itg.be/WPshsop.

[26] Y. Souteyrand, M. A. Banda, J. Kaufman, J. H. Perriëns, and D. Yu, "Investment in HIV/AIDS programs: does it help strengthen health systems in developing countries?" Globalization and Health, vol. 4, article 8, 2008.

[27] E. Wouters, W. Van Damme, D. van Rensburg, C. Masquillier, and H. Meulemans, "Impact of community-based support services on antiretroviral treatment programme delivery and outcomes in resource-limited countries: a synthetic review," BMC Health Services Research, vol. 12, article 194, 2012.

[28] M. W. G. Brinkhof, M. Pujades-Rodriguez, and M. Egger, "Mortality of patients lost to follow-up in antiretroviral treatment programmes in resource-limited settings: systematic review and meta-analysis," PLoS ONE, vol. 4, no. 6, Article ID e5790, 2009. 
[29] M. Egger, B. D. Spycher, J. Sidle et al., "Correcting mortality for loss to follow-up: a nomogram applied to antiretroviral treatment programmes in sub-Saharan Africa," PLoS Medicine, vol. 8, no. 1, Article ID e1000390, 2011.

[30] J. K. Yu, S. C. Chen, K. Y. Wang et al., "True outcomes for patients on antiretroviral therapy who are "lost to follow-up" in Malawi," Bull World Health Organ, vol. 85, no. 7, pp. 550-554, 2007.

[31] A. D. Harries, R. Zachariah, S. D. Lawn, and S. Rosen, "Strategies to improve patient retention on antiretroviral therapy in sub-Saharan Africa," Tropical Medicine and International Health, vol. 15, no. 1, pp. 70-75, 2010.

[32] M. Maruza, M. F. P. Militão Albuquerque, I. Coimbra et al., "Risk factors for default from tuberculosis treatment in HIVinfected individuals in the state of Pernambuco, Brazil: a prospective cohort study," BMC Infectious Diseases, vol. 11, article 351, 2011.

[33] K. Mungrue, A. Beharry, J. Kalloo et al., "Trends in HIV/TB coinfection in Trinidad and Tobago for the period 1998-2007," Journal of the International Association of Physicians in AIDS Care, vol. 8, no. 3, pp. 170-175, 2009.

[34] WHO, "Antiretroviral therapy for hiv infection in infants and children: towards universal access," Recommendations for a public health approach, 2010, http://www.who.int/hiv/pub/ paediatric/infants2010/en/index.html.

[35] WHO, "Consolidated guidelines on the use of antiretroviral drugs for treating and preventing HIV infection," Recommendations for a public health approach, 2013, http://apps.who.int/ iris/bitstream/10665/85321/1/9789241505727_eng.pdf.

[36] G. Tembo, H. Friesan, G. Asiimwe-Okiror et al., "Bed occupancy due to HIV/AIDS in an urban hospital medical ward in Uganda," AIDS, vol. 8, no. 8, pp. 1169-1171, 1994.

[37] M. Fabiani, S. Accorsi, R. Aleni et al., "Estimating HIV prevalence and the impact of HIV/AIDS on a Ugandan hospital by combining serosurvey data and hospital discharge records," Journal of Acquired Immune Deficiency Syndromes, vol. 34, no. 1, pp. 62-66, 2003.

[38] A. Buvé, "AIDS and hospital bed occupancy: an overview," Tropical Medicine and International Health, vol. 2, no. 2, pp. 136139, 1997.

[39] G. Arthur, S. M. Bhatt, D. Muhindi, G. A. Achiya, S. M. Kariuki, and C. F. Gilks, "The changing impact of HIV/AIDS on Kenyatta National Hospital, Nairobi from 1988/89 through 1992 to 1997," AIDS, vol. 14, no. 11, pp. 1625-1631, 2000.

[40] T. Puthanakit, L. Aurpibul, P. Oberdorfer et al., "Hospitalization and mortality among HIV-infected children after receiving highly active antiretroviral therapy," Clinical Infectious Diseases, vol. 44, no. 4, pp. 599-604, 2007.

[41] R. M. Viani, M. R. G. Araneta, J. G. Deville, and S. A. Spector, "Decrease in hospitalization and mortality rates among children with perinatally acquired HIV type 1 infection receiving highly active antiretroviral therapy," Clinical Infectious Diseases, vol. 39, no. 5, pp. 725-731, 2004.

[42] D. M. Gibb, T. Duong, P. A. Tookey et al., "Decline in mortality, AIDS, and hospital admissions in perinatally HIV-1 infected children in the United Kingdom and Ireland," British Medical Journal, vol. 327, no. 7422, pp. 1019-1023, 2003.

[43] K. Kober and W. Van Damme, Expert Patients And AIDS Care: A Literature Review on Expert Patient Programmes in High-Income Countries, and an Exploration of Their Relevance for HIV/AIDS Care in Low-Income Countries with Severe Human Resource
Shortages, Institute of Tropical Medicine, Antwerp, Belgium, 2006.

[44] E. F. Nsutebu, J. D. Walley, E. Mataka, and C. F. Simon, "Scalingup HIV/AIDS and TB home-based care: lessons from Zambia," Health Policy and Planning, vol. 16, no. 3, pp. 240-247, 2001.

[45] B. Tewodros, R. Freya, A. Yibeltal, B. Atakilti, and W. Van Damme, "Disease control programs contribution to Health System Strengthening: good practices and new approaches for scale up," Working Paper Series of the Studies in Health Services Organisation and Policy 4, Federal Ministry of Health, Ethiopia and the Institute of Tropical Medicine, Antwerp, Belgium, 2011.

[46] WHO, "WHO Global Health Sector Strategy on HIV/AIDS, 2011-2015," 2011, http://whqlibdoc.who.int/publications/2011/ 9789241501651_eng.pdf.

[47] C. Potter and R. Brough, "Systemic capacity building: A hierarchy of needs," Health Policy and Planning, vol. 19, no. 5, pp. 336-345, 2004.

[48] F. Rasschaert, M. Pirard, M. P. Philips et al., "Positive spill-over effects of ART scale up on wider health systems development: Evidence from Ethiopia and Malawi," Journal of the International AIDS Society, vol. 14, supplement 1, article s3, 2011.

[49] R. Brugha, J. Simbaya, A. Walsh, P. Dicker, and P. Ndubani, "How HIV/AIDS scale-up has impacted on non- HIV priority services in Zambia," BMC Public Health, vol. 10, article 540, 2010. 


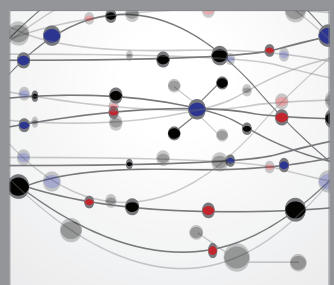

The Scientific World Journal
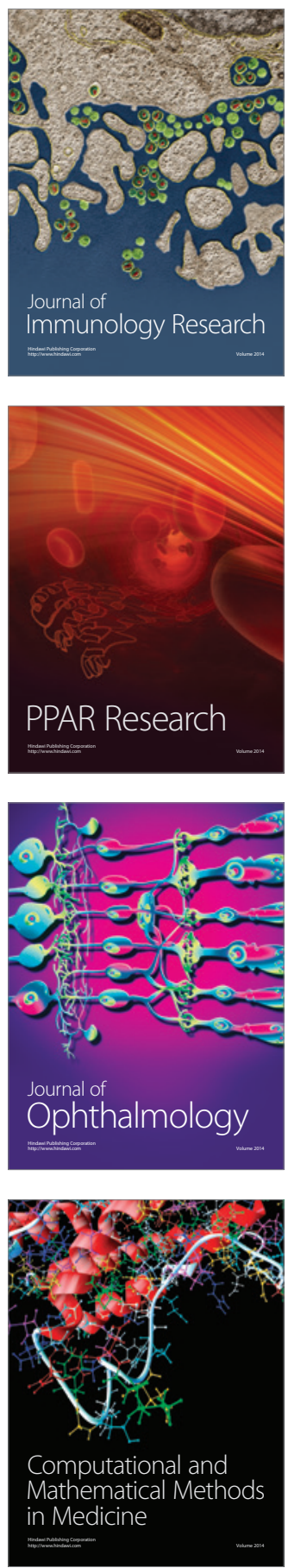

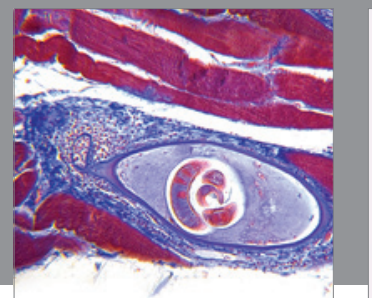

Gastroenterology

Research and Practice
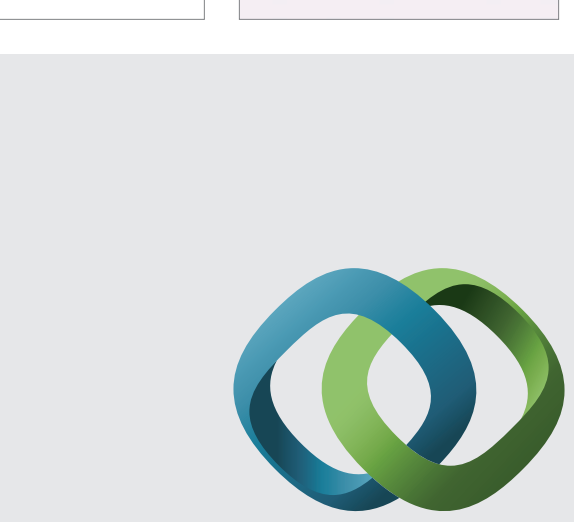

\section{Hindawi}

Submit your manuscripts at

http://www.hindawi.com
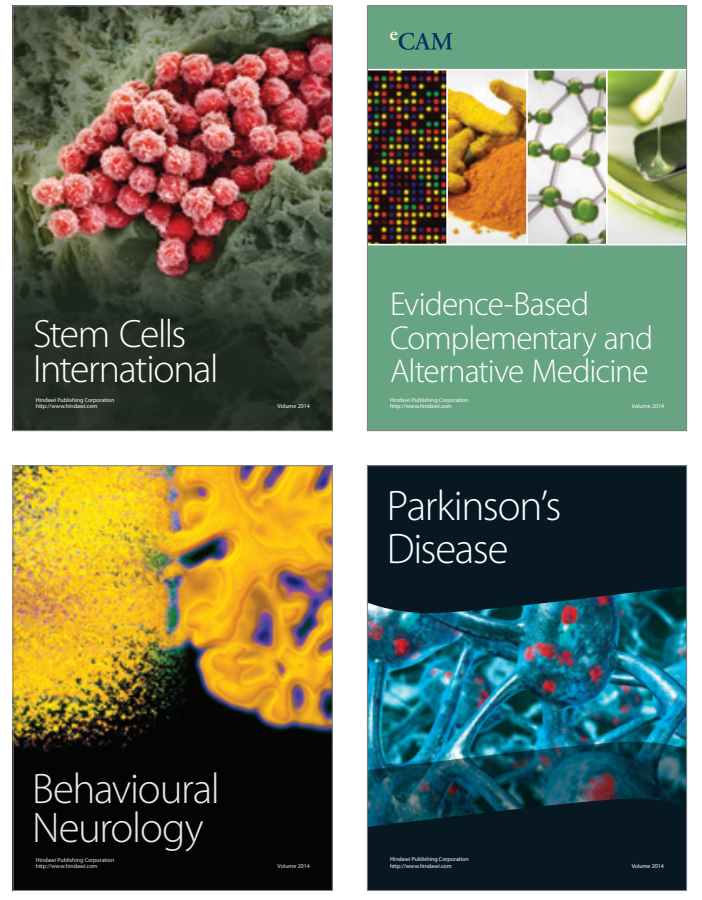
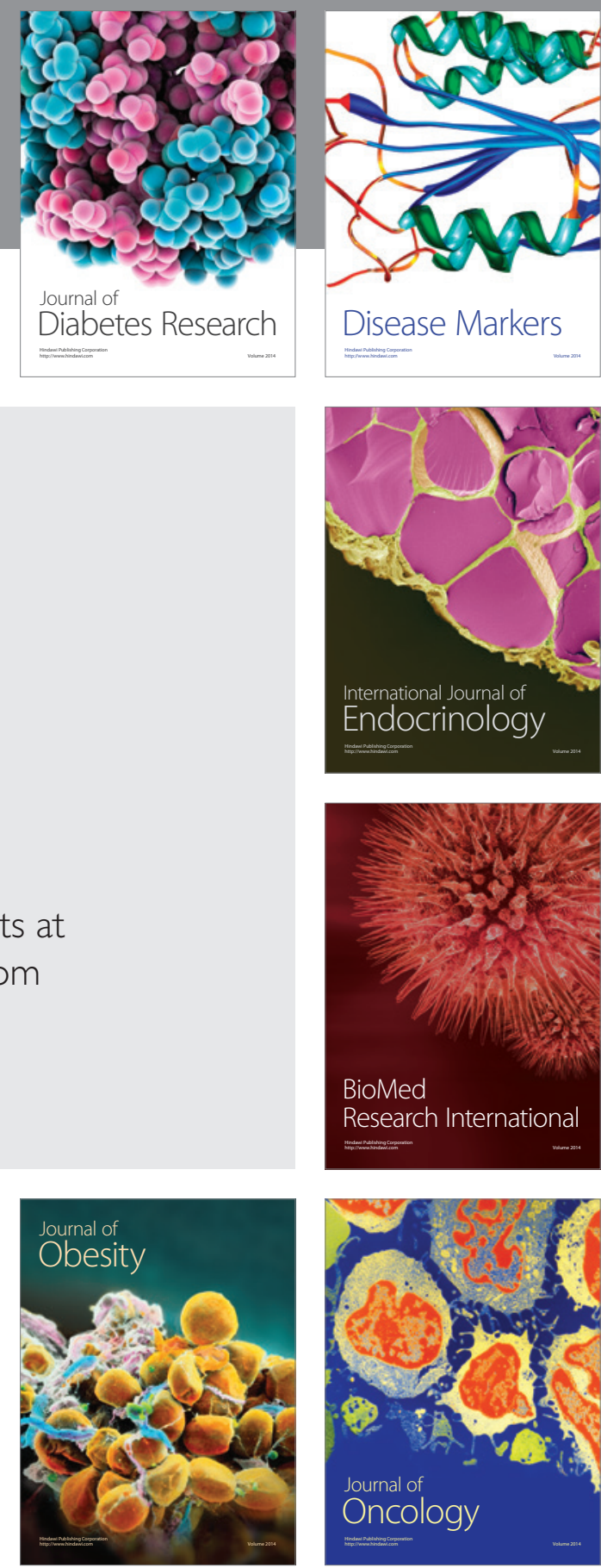

Disease Markers
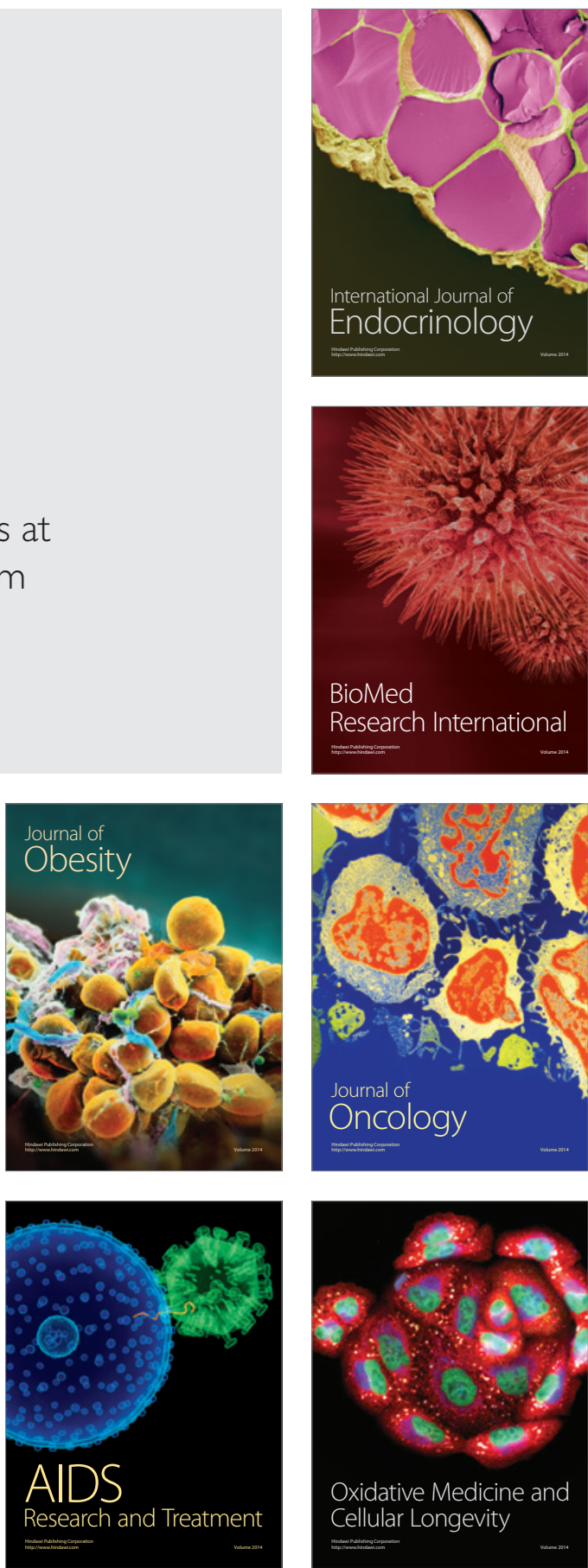